\title{
Establishing a patient registry study database of dust mite allergic asthma in children: design, methodology and preliminary exploration
}

\begin{abstract}
Juan Zhou ${ }^{1,2,3}$, Weiguo Li ${ }^{1,2}$, Xiang Wen ${ }^{1,2}$, Dan Zeng ${ }^{1,2}$, Jilei Lin ${ }^{1,2}$, Shiyi Chen ${ }^{2,4}$, Na Zang ${ }^{1,2}$, Yu Deng ${ }^{1,2}$, Xiaohong Xie ${ }^{1,2}$, Luo Ren ${ }^{2,4}$, Enmei Liu ${ }^{1,2}$

${ }^{1}$ Department of Respiratory Medicine, Children's Hospital of Chongqing Medical University, National Clinical Research Center for Child Health and Disorders, Ministry of Education Key Laboratory of Child Development and Disorders, Chongqing, China; ${ }^{2}$ Chongqing Key Laboratory of Pediatrics, Chongqing, China; ${ }^{3}$ Department of Pediatrics, Guizhou Provincial People's Hospital, Medical College of Guizhou University, Guiyang, China; ${ }^{4}$ Pediatric Research Institute Children's Hospital of Chongqing Medical University, National Clinical Research Center for Child Health and Disorders, Ministry of Education Key Laboratory of Child Development and Disorders, Chongqing, China

Contributions: (I) Conception and design: E Liu, L Ren; (II) Administrative support: E Liu; (III) Provision of study materials or patients: E Liu; (IV) Collection and assembly of data: J Zhou, W Li, X Wen, D Zeng, S Chen; (V) Data analysis and interpretation: J Zhou, L Ren, J Lin, N Zang, Y Deng, X Xie; (VI) Manuscript writing: All authors; (VII) Final approval of manuscript: All authors.

Correspondence to: Luo Ren, PhD. Pediatric Research Institute Children's Hospital of Chongqing Medical University, National Clinical Research Center for Child Health and Disorders, Ministry of Education Key Laboratory of Child Development and Disorders, China International Science and Technology Cooperation Base of Child Development and Critical Disorders, Chongqing Key Laboratory of Pediatrics, Chongqing, China. Email: renluo@yeah.net; Enmei Liu, MD, PhD. Department of Respiratory Medicine Children’s Hospital of Chongqing Medical University, National Clinical Research Center for Child Health and Disorders, Ministry of Education Key Laboratory of Child Development and Disorders, China International Science and Technology Cooperation Base of Child Development and Critical Disorders, Chongqing Key Laboratory of Pediatrics, Chongqing, China. No. 136 Zhongshan 2nd Road, Yuzhong District, Chongqing, China. Email: emliu186@126.com.
\end{abstract}

Background: Asthma is a heterogeneous disease with different phenotypes, endotypes and responses to treatment. Dust mite allergic asthma (DMAA) is the most common type in children. Compared with randomized control trials, a patient registry study (PRS) can reflect the real physical condition and clinical diagnosis more comprehensively.

Methods: Children who visited the asthma clinic of the Children's Hospital of Chongqing Medical University between August 2018 and August 2020, and met the inclusion criteria and also agreed to participate, were enrolled in the registry study. Clinical information, laboratory tests and peripheral blood samples were collected after informed consent was given by guardians.

Results: To date, 208 children have been enrolled in the patient registry database of DMAA. They are mainly male, with $>50 \%$ having a history of allergic rhinitis, cesarean section, positive family history and passive smoking. Eosinophils and total immunoglobulin $\mathrm{E}$ levels were all significantly higher than normal. According to results for the childhood asthma control test (c-ACT) and ratio of forced expiratory volume in $1 \mathrm{~s}$ to predicted value after inhaled corticosteroid treatment, the uncontrolled group had higher hemoglobin $(\mathrm{Hb})$ levels than the control group. The group exhibiting abnormal pulmonary function was older, and had longer disease duration, higher fractional exhaled nitric oxide and $\mathrm{Hb}$ than the group in which pulmonary function was restored.

Conclusions: We have preliminarily established a registered study database of children with DMAA. By cluster analysis and using blood samples, we can further study the different pathophysiological mechanisms in order to provide more individualized and targeted treatments for all children.

Keywords: Allergic asthma; children; dust mites; registry study database

Submitted Apr 27, 2021. Accepted for publication Jun 15, 2021.

doi: $10.21037 / \mathrm{atm}-21-2566$

View this article at: https://dx.doi.org/10.21037/atm-21-2566 


\section{Introduction}

There are about 300 million patients with asthma worldwide. Over the past 20 years, both the prevalence and severity of asthma in children have continuously increased (1). Asthma usually starts from childhood (2), and is the most common chronic inflammatory disease of the respiratory tract in children. Symptoms are usually aggravated in the morning or at night (3), with the main clinical manifestations being recurrent wheezing, cough, and shortness of breath. Because of its recurrent nature, asthma seriously damages the physical and mental health of children, which increases the burden on both the family and society. According to the Global Burden of Disease 2010, asthma has become one of the 20 high-burden diseases, with a contribution rate $>1 \%$ to the disability-adjusted life year (4).

Allergic asthma is the most common type of asthma in children, with dust mites, pet dander, fungi and other substances being the main risk factors (5). In countries other than China, $65 \%$ of children with asthma are allergic to dust mites, and in the domestic cohort it is $85 \%(6,7)$. Dust mite allergens can activate mast cells and $\mathrm{T}$ cells directly and indirectly, which result in inflammatory damage of airway epithelium (8). There is also a synergistic effect with viruses $(6,9)$, thus affecting the lung function and long-term respiratory health of children $(10,11)$.

Asthma is a heterogeneous disease with diverse clinical features (phenotypes), pathophysiological mechanisms (endotypes) and drug responses (12). As early as 2002, it was reported that $45 \%$ of asthmatic children had a poor response to inhaled corticosteroids (ICS) (13). A 50 -year follow-up birth cohort study found that the severity and heterogeneity of childhood asthma could lead to different clinical outcomes, making the remission rate from childhood asthma to adulthood only $15 \%$ (14). Gaining further knowledge about the different pathogenesis and treatment responses of childhood asthma is beneficial to early control of airway inflammation, improvement of lung function, and prevention of persistence of asthma symptoms in children.

Real-world clinical research has received increasing attention in recent years (15), of which patient registry studies (PRS) are an important part. By this method, the patient's health information is registered to evaluate disease status or the outcome of exposure. Compared with the classical randomized control trial, which strictly controls the inclusion, randomization and intervention, the selection of diagnosis and treatment in PRS completely depends on the disease and in the case of children, their wishes and those of their guardians. By using a PRS, we can more comprehensively reflect the real physical condition, clinical diagnosis, and treatment process of children with asthma.

Although there are some large databases related to population medical insurance which containing children's data, and databases related to children's nervous system (16-18), these databases only get clinical data of patients and can only perform retrospective research when encountering clinical problems. We planned to establish a PRS database of dust mite allergic asthma (DMAA) in children, which would contain the children's clinical information, laboratory indicators, and peripheral blood samples. Further analysis of these data would then enable us to research the different phenotypes, endotypes and causes of different reactions to treatments, in order to implement standardized and individualized treatment that improves the prognosis and quality of life. We present the following article in accordance with the STROBE reporting checklist (available at https://dx.doi.org/10.21037/atm-21-2566).

\section{Methods}

\section{Research method}

We established the database according to an established method of developing patient registry databases (19). The research process (Figure 1) had the following stages. (I) Determine research objectives: to study the heterogeneity of asthma and provide precise treatment. (II) Identify the target population: children with DMAA. (III) Ethics approval: the study was approved by the Institutional Review Board of the Children's Hospital of Chongqing Medical University (File No. 2018-02, 2019-27-1). (IV) Experiment registration: the study protocol was registered in the Chinese Clinical Trials Registry (Registration No. ChiCTR2000038778). (V) Create study protocol: items, methods, data collection and storage; collection, processing, utilization and storage of blood samples; follow-up. (VI) Protocol implementation and improvement. All procedures performed in this study involving human participants were in accordance with the Declaration of Helsinki (as revised in 2013).

\section{Study population}

Children with DMAA, who visited the asthma clinic of 


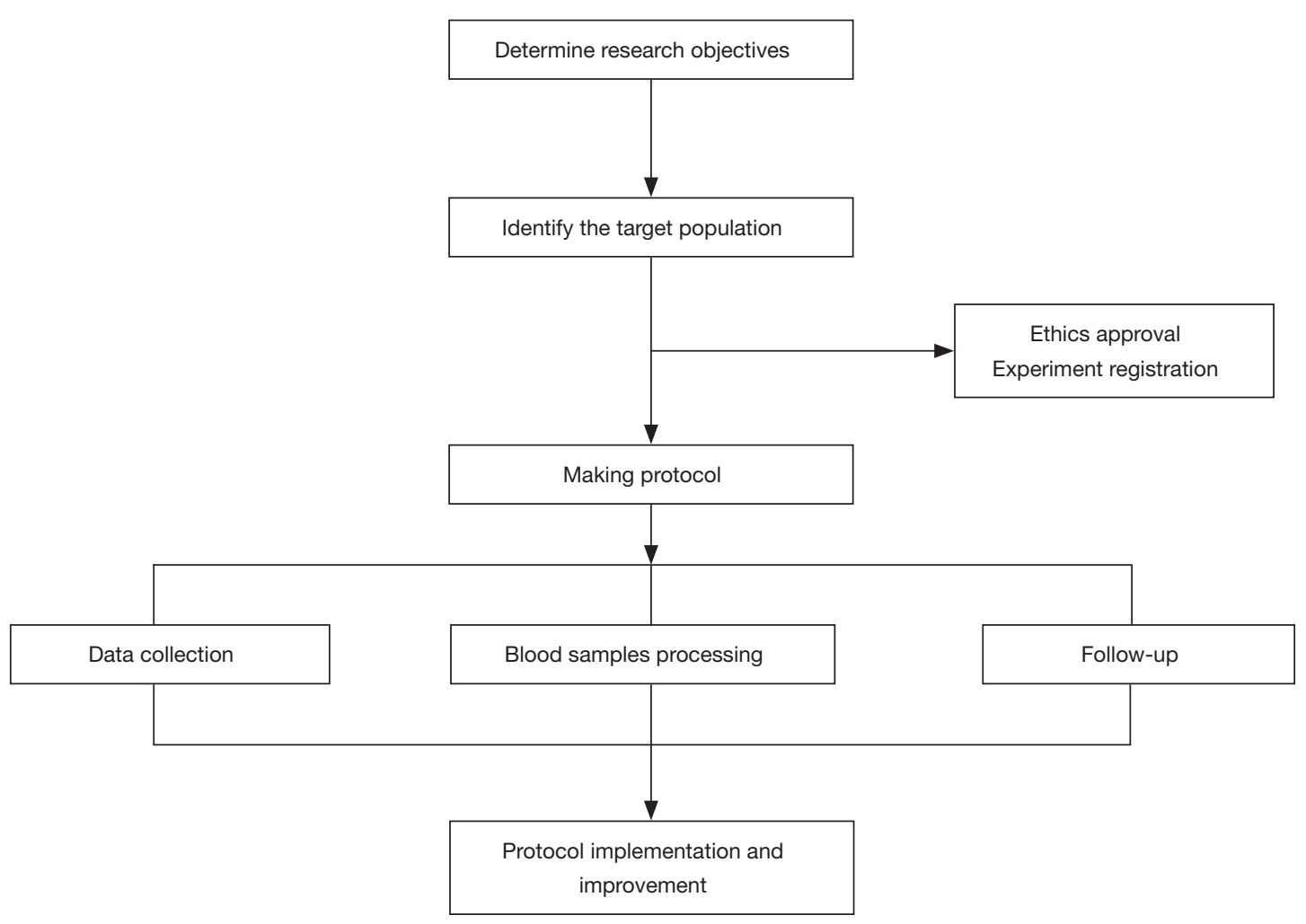

Figure 1 Flow-sheet of the research process.

the Children's Hospital of Chongqing Medical University between August 2018 and August 2020, comprised the study registry population, provided they met the inclusion criteria: (I) met the diagnostic criteria of the Global Initiative for Asthma guidelines (http://ginasthma.org/); (II) age in the range of 6 to 12 years; (III) positive skin prick test to Dermatophagoides pteronyssinus (Der $\mathrm{p}$ ) or D. farina (Der $\mathrm{f}$ ) of at least "+++". The children were also free of the following exclusion criteria: (I) heart, lung, liver, kidney, or other organic diseases; (II) bronchial foreign bodies, tuberculosis or other lung diseases. Following informed consent from the statutory guardian, the children were enrolled in the database.

\section{Data collection}

At the outpatient visit, clinical consultation, auxiliary examination and peripheral blood sample collection were performed. Clinical information was collected by a trained physician with a detailed questionnaire (Appendix 1). The information was entered and stored in Microsoft Excel spreadsheets, with the physician and project leader each keeping a copy as backup. The auxiliary examination included routine blood, lung function [including FEV1\% (ratio of forced expiratory volume in 1 $\mathrm{s}$ to predicted value); Master Screen JAEGER, Germany], and fractional exhaled nitric oxide (FeNO) (NIOX MINO, Sweden) measurements.

\section{Blood sample collection and processing}

Collection: Fresh blood (2-3 mL) and serum were collected in EDTA-coated anticoagulant tube and pro-coagulant tube respectively, before being transported to the laboratory at low temperature.

Processing: (I) the pro-coagulant tubes were centrifuged at $1,000 \mathrm{~g}$ at $4{ }^{\circ} \mathrm{C}$ for $5 \mathrm{~min}$. The top $500 \mu \mathrm{L}$ of serum was sent for measurement of total immunoglobulin $\mathrm{E}(\mathrm{t} \operatorname{IgE})$ and dust mite specific IgE (sIgE; ImmunoCAP-100 ${ }^{\circledR}$, Thermo Fisher Scientific, Sweden), and the remainder was stored at $-80{ }^{\circ} \mathrm{C}$. (II) The anticoagulant tubes were centrifuged at $800 \mathrm{~g}$ at $4{ }^{\circ} \mathrm{C}$ for $5 \mathrm{~min}$, and the plasma was stored at $-80{ }^{\circ} \mathrm{C}$. The remaining cells were centrifuged in a FicollPaque density gradient, and the layer of peripheral blood 
mononuclear cells (PBMC) was collected and stored at $-80^{\circ} \mathrm{C}$.

\section{Blood sample utilization and storage}

(I) Serum samples collected between August 2018 and February 2019 were tested for interleukin (IL)-4, -5 and -13 , and periostin by ELISA (Neobioscience, Wuhan, China). The remaining serum, plasma and PBMC were stored at $-80^{\circ} \mathrm{C}$ in the laboratory.

(II) Starting from November 2019, the sera, plasma and PBMC were stored in the biological sample bank (biobank) of the Children's Hospital of Chongqing Medical University. Each biobank tube had a unique identification code and baseline information, which included the identification code, and the child's name, age, sex, and sample type, was entered in the online information management system (ebiobank). Each tube also had a unique storage location.

\section{Follow-up}

After 1 year of treatment, medication, childhood asthma control test (c-ACT), routine blood tests, lung function, FEV1\%, $\operatorname{tgE}$ and dust mite $\operatorname{sgE}$ were reassessed, and peripheral blood was collected on the day of the second visit for follow-up study.

\section{Statistical analysis}

SPSS24.0 software (Chicago, IL, USA) was used for statistical analysis. All continuous data are described as medians with interquartile ranges or as means with standard deviation. Categorical data are presented as proportions or percentages. Continuous data between groups were compared using the $t$-test for normally distributed variables or the Mann-Whitney test for abnormally distributed variables. The $\chi^{2}$ test was used for categorical variables $(\mathrm{P}<0.05$ was considered statistically significant). Deletion method is used to deal with missing data.

\section{Results}

\section{Baseline database information}

A total of 208 cases were enrolled between August 2018 and August 2020, based on the inclusion and exclusion criteria. Clinical information, peripheral blood samples, routine blood tests, FeNO and IgE measurements were collected from all the children. FEV1\% was calculated for 194 cases; IL-4 was tested in 86 cases, IL-13 in 92 cases, and IL-5 in 95 cases; periostin was tested in 58 cases.

\section{Clinical information}

The ratio of male to female children was 135:73, the median age was 8 years, and the median course of disease was 3 years. A majority of the patients $(90.58 \%)$ had complications of allergic rhinitis (AR), $56.25 \%$ had a positive family history; $51.44 \%$ had cigarette smoke exposure, and $71.15 \%$ were receiving ICS treatment (Table 1).

\section{Laboratory tests}

The median of $\operatorname{tgE}$ was $471.00 \mathrm{kU} / \mathrm{L}$, the ratios of Der p sIgE/tIgE and Der f sIgE/tIgE were $12.39 \%$ and $14.80 \%$, respectively, and the median of $\mathrm{E} \%$ (percentage of eosinophils) was $7 \%$. All values were higher than normal. Due to the different test kits and conditions used in different laboratories, there is no recognized standard for IL-4, IL-13, IL-5 and periostin, and only the test data are listed (Table 2).

\section{Comparison between groups according to post-treatment c-ACT score}

Based on the post-treatment c-ACT scores, the children were divided into asthma uncontrolled group ( $\leq 19$ points) and asthma controlled group (24-27 points). There were 94 cases in the two groups combined, which included 21 cases $(22.34 \%)$ in the uncontrolled group and 73 cases $(77.66 \%)$ in the controlled group. Comparison of the clinical and laboratory indicators of the two groups showed that hemoglobin $(\mathrm{Hb})$ in the uncontrolled asthma group was higher than in the controlled asthma group $(\mathrm{P}<0.05)$ (Table 3).

\section{Comparison between groups according to post-treatment FEV 1\%}

Following treatment, the children with FEV1\% $<80 \%$ were classified as the pulmonary function abnormality group, and those with FEV1\% $>80 \%$ were classified as the pulmonary function restored group. There were 141 children in the two groups combined: 20 cases $(14.84 \%)$ in the abnormality group and 121 cases $(85.82 \%)$ in the restored group. 
Table 1 Demographic characteristics and clinically relevant information of pediatric asthma patients

\begin{tabular}{lc}
\hline Characteristic & Patients $(\mathrm{n}=208)$ \\
\hline Male: female & $135: 73$ \\
Age (years) & $8[7,10]$ \\
Duration of illness (years) & $3[2,5]$ \\
History of allergic rhinitis, $\mathrm{n}(\%)$ & $188(90.38)$ \\
Family history of allergy or asthma, $\mathrm{n}(\%)$ & $117(56.25)$ \\
Cigarette smoke exposure, $\mathrm{n}(\%)$ & $107(51.44)$ \\
Premature delivery, $\mathrm{n}(\%)$ & $19(9.13)$ \\
Delivery by cesarean section, $\mathrm{n}(\%)$ & $146(70.19)$ \\
Glucocorticoid treatment, $\mathrm{n}(\%)$ & \\
Never used or discontinued & $58(27.88)$ \\
ICS & $45(21.63)$ \\
ICS + LABA & $105(50.48)$ \\
Allergen immunotherapy, $\mathrm{n}(\%)$ & $32(15.39)$ \\
Never used & $146(70.19)$ \\
In use & $140(67.31)$ \\
Previous use & $43(20.67)$ \\
C-ACT, $\mathrm{n}(\%)$ & $25(12.02)$ \\
Uncontrolled asthma ( $\leq 19)$ & \\
Partly controlled asthma $(\geq 20$ and $\leq 23)$ & \\
Controlled asthma $(\geq 24$ and $\leq 27)$ & \\
\hline
\end{tabular}

ICS, inhaled corticosteroids; LABA, long-acting $\beta 2$ adrenergic agonists; C-ACT, childhood asthma control test. BMI, body mass index.

Comparison of the clinical and laboratory indicators of the two groups showed that the patients in the abnormality group were older, had a longer course of disease, and higher $\mathrm{FeNO}$ and $\mathrm{Hb}$ than those in the restored group $(\mathrm{P}<0.05)$. There was no statistical difference in the remaining indicators (Table 4).

\section{Discussion}

Here we present our method of creating a PRS database of
Table 2 Laboratory indicators of pediatric asthma patients

\begin{tabular}{lc}
\hline Items & Patients $(\mathrm{n}=208)$ \\
\hline tlgE $(\mathrm{Ku} / \mathrm{L})$ & $471.00(190.75,844.00)$ \\
Der f slgE/tlgE (\%) & $12.39(8.61,18.36)$ \\
Der p slgE/tlgE (\%) & $14.80(9.70,19.98)$ \\
Eos\% & $7.00(4.13,9.75)$ \\
FEV1\% & $94.22 \pm 12.41$ \\
FeNO $(\mathrm{ppb})$ & $24.30(12.60,55.18)$ \\
PLT $\left(\times 10^{9} / \mathrm{L}\right)$ & $293.74 \pm 76.75$ \\
Hb $(\mathrm{g} / \mathrm{L})$ & $132.35 \pm 9.88$ \\
$\mathrm{IL}-4(\mathrm{pg} / \mathrm{mL})$ & $4.79(3.21,6.68)$ \\
$\mathrm{IL}-13(\mathrm{pg} / \mathrm{mL})$ & $125.47(69.16,227.12)$ \\
$\mathrm{IL}-5(\mathrm{pg} / \mathrm{mL})$ & $23.64(20.54,26.78)$ \\
Periostin $(\mathrm{ng} / \mathrm{mL})$ & $319.71 \pm 38.19$ \\
\hline
\end{tabular}

tlgE, total IgE; Der $\mathrm{p}$ slgE/tlgE (\%), ratio of Dermatophagoides pteronyssinus specific $\lg \mathrm{E}$ to total $\lg \mathrm{E}$; Der $\mathrm{f}$ slgE/tlgE, ratio of Dermatophagoides farina specific IgE to total IgE; Eos\%, percentage of eosinophils; FEV1\%, ratio of forced expiratory volume in $1 \mathrm{~s}$ to predicted value; FeNO, fractional exhaled nitric oxide; PLT, platelets; Hb, hemoglobin; IL, interleukin.

DMAA in children and an outline of the current operation of the database. The existing data were preliminary explored according to the post-treatment c-ACT score and FEV1\% results to guide the next direction of research. At present, 208 children have been enrolled, and the sample size will be expanded in the future. The database includes clinical information, laboratory indicators, and serum, plasma, and PBMC samples.

Preliminary analysis of the database showed that, similar to previous studies elsewhere (20-25), the children with DMAA were mainly male, cesarean section was the main mode of birth, and a positive family history and passive smoking history existed in more than half of the cases. As reported (26), there is a strong correlation between allergic asthma and AR. In our database, the total prevalence of AR reached $90.38 \%$. Reducing allergen exposure is one of the most important measures to control and treat AR and asthma, which can improve the symptoms of asthma and rhinitis. Currently, measures to reduce HDM exposure include keeping room humidity below $50 \%$, wrapping mattresses and pillows with impervious covers, regularly cleaning bedding with hot water, removing carpets and plush toys, and regularly using high-efficiency particulate 
Table 3 Comparison of uncontrolled and controlled asthma groups

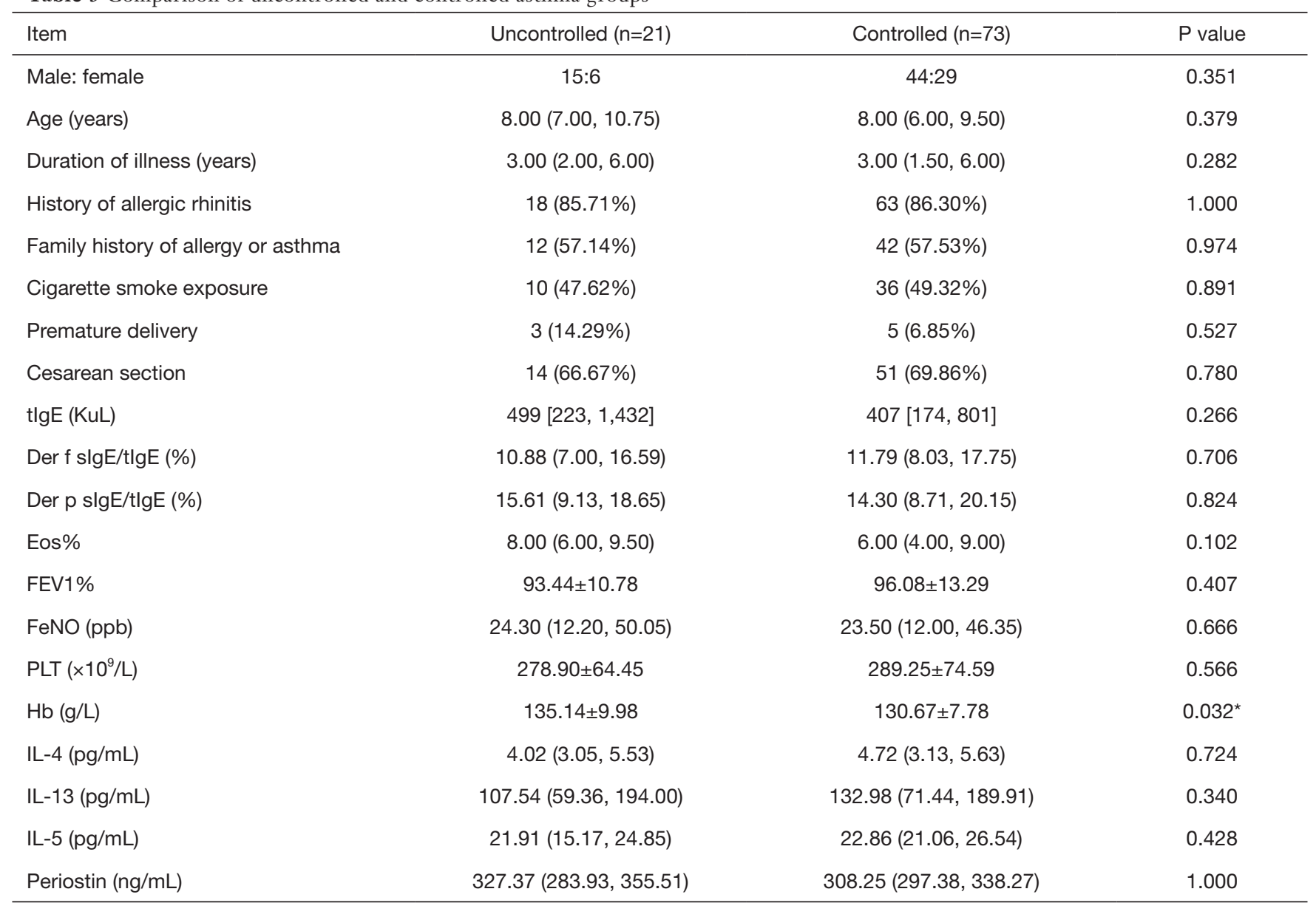

${ }^{*} \mathrm{P}<0.05$. tlgE, total $\lg \mathrm{E}$; Der $\mathrm{p}$ slgE/tlgE (\%), ratio of Dermatophagoides pteronyssinus specific $\lg \mathrm{E}$ to total $\lg \mathrm{E} ; \mathrm{Der} f$ slgE/tlgE, ratio of Dermatophagoides farina specific IgE to total IgE; Eos\%, percentage of eosinophils; FEV1\%, ratio of forced expiratory volume in $1 \mathrm{~s}$ to predicted value; FeNO, fractional exhaled nitric oxide; PLT, platelets; Hb, hemoglobin; IL, interleukin.

air filters and suicide agents $(27,28)$. In terms of laboratory indicators, eosinophils, $\operatorname{tgE}$ and dust mite $\operatorname{sgE} / \mathrm{t} \operatorname{IgE}$ were all significantly higher than normal. It has been reported (29) that children with heterogeneous diseases (including asthma and eczema) are more prone to anemia, but this correlation was has not found in our data. According to the post-treatment c-ACT score and $\mathrm{FEV} 1 \%, \mathrm{Hb}$ in the uncontrolled group and the pulmonary function abnormality group was higher. Further studies are needed to determine whether this was simply due to the increase of $\mathrm{Hb}$ synthesis caused by hypoxia or related to other factors, such as expression changes of haptoglobin (related to $\mathrm{Hb}$ catabolism), which plays an important role in immune regulation and the pulmonary inflammatory response (30). The age, disease course and FeNO levels in the pulmonary function abnormality group were greater than those in the pulmonary function restored group, which is consistent with previous reports $(31,32)$. Kanemitsu et al. (33) reported that periostin was an independent risk factor for FEV1 decline. However, in our current data, periostin showed no difference between the pulmonary function abnormality and pulmonary function restored groups after treatment, which may be related to the different populations and fewer cases measured. The causes of different responses to treatment could not be determined based on the present results of c-ACT and FEV1\% after treatment. Considering the heterogeneous characteristics of asthma, further cluster analysis is clearly needed. In addition to the common Th2 inflammatory factors, interferon- $\gamma$ and IL-17 should also be investigated in order to understand whether there are other inflammatory cell types or mechanisms. Finally, the genetic information and 
Table 4 Comparison of pulmonary function abnormality and restored groups

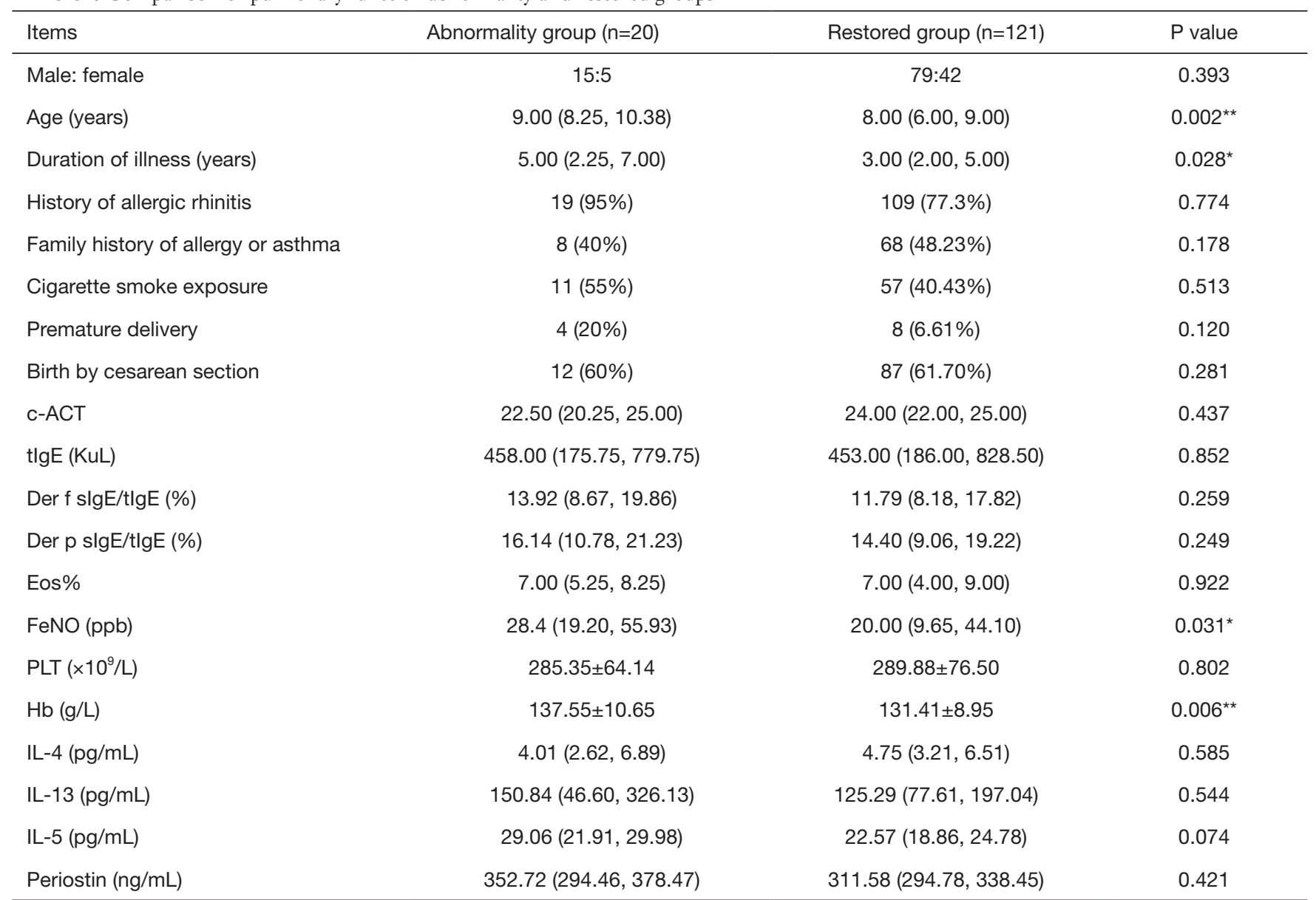

${ }^{*} \mathrm{P}<0.05$, ${ }^{\star} \mathrm{P}<0.01$. tlgE, total IgE; Der $\mathrm{p}$ slgE/tlgE (\%), ratio of Dermatophagoides pteronyssinus specific IgE to total IgE; Der $\mathrm{f}$ slgE/ tlgE, ratio of Dermatophagoides farina specific IgE to total IgE; Eos\%, percentage of eosinophils; FEV1\%, ratio of forced expiratory volume in $1 \mathrm{~s}$ to predicted value; FeNO, fractional exhaled nitric oxide; PLT, platelets; Hb, hemoglobin; IL, interleukin.

transcriptome of the children with different treatment responses could be studied to further delve into the pathophysiological mechanism.

Multiple investigations of childhood asthma have been conducted in China and abroad. In contrast with most other studies, our study design offered the opportunity of crosssectional analysis, as well as retrospective and prospective investigations of the database. At the same time, in contrast with other databases $(17,18)$, we not only collected and archived the clinical information but also stored the peripheral blood samples of the children, which allows for future clustering analysis of clinical information as well as selection of questions of interest to further explore the relevant mechanisms.

In the process of database creation, we also summarized the insufficiencies and developed methods of improvement. (I) In the early stages, the collected serum, plasma and PBMC were frozen at $-80{ }^{\circ} \mathrm{C}$ in a laboratory refrigerator, and the samples had no designated storage location, which created difficulties with timely retrieval of samples. After November 2019, the corresponding serum, plasma and PBMC samples have been stored in the biological sample library of the hospital and can be efficiently located by inputting the name of the patient. (II) Initially, in order to understand the basic inflammatory state of children with DMAA, inflammatory factors such as IL-4, IL-5 and IL-13 in serum were detected, which consumed a large number of samples. After March 2019, however, these factors were no longer routinely detected, because the samples in the biological sample bank could be accessed for any future 
testing or research needs. (III) As regards ethics, we started with an age threshold of 6-12 years old. In the clinical course, however, it was found that although standard preventive treatment had been used, the effect on many children was still not ideal. This part of children with poor effect were mainly adolescent children, consistent with other studies (34). We will, therefore, extend the age range to 18 years old in the future work. (IV) It was originally planned to follow-up after 1 year, but in the actual implementation process, the follow-up rate was low due to lack of subsequent visits and reluctance of family members to allow blood donation again. In order to improve the follow-up rate, we plan to adjust the follow-up time to three times, which would be 3-4 months, $6-8$ months, and 1 year after treatment. (V) At present, our data are from a single center, and thus there is potential for some bias. If conditions permit, we will cooperate with other hospitals and other regions to reduce bias through multicenter research.

\section{Conclusions}

We have established a proof-of-concept database of children with DMAA. The database will continue to be expanded and improved. Through cluster analysis of children with similar phenotypes, and further research on genetics, epigenetics and molecular mechanisms using the clinical blood samples in the database, we should better understand the different pathophysiology of childhood asthma, find ways to improve the treatment effect, and provide more individualized and targeted treatments. After this initial exploration, the information collection, sample processing and storage methods have been standardized, which should provide a solid clinical basis for further research into the mechanism of allergic asthma.

\section{Acknowledgments}

Our thanks to Professor Sun Xin of West China Hospital of Sichuan University for help and guidance.

Funding: This study was sponsored by National Clinical Research Center for Child Health and Disorders (grant No. NCRC-2019-GP-06); Chongqing Municipal Science and Technology Bureau (The Central Government Guides Development of Local Science and Technology Fund); Clinical Research Center of Children's Hospital of Chongqing Medical University.

\section{Footnote}

Reporting Checklist: The authors have completed the STROBE reporting checklist. Available at https://dx.doi. org/10.21037/atm-21-2566

Data Sharing Statement: Available at https://dx.doi. org/10.21037/atm-21-2566

Conflicts of Interest: All authors have completed the ICMJE uniform disclosure form (available at https://dx.doi. org/10.21037/atm-21-2566). All authors report that this study was sponsored by National Clinical Research Center for Child Health and Disorders (grant No. NCRC-2019GP-06); Chongqing Municipal Science and Technology Bureau (The Central Government Guides Development of Local Science and Technology Fund); Clinical Research Center of Children's Hospital of Chongqing Medical University. The authors have no other conflicts of interest to declare.

Ethical Statement: The authors are accountable for all aspects of the work in ensuring that questions related to the accuracy or integrity of any part of the work are appropriately investigated and resolved. All procedures performed in this study involving human participants were in accordance with the Declaration of Helsinki (as revised in 2013). The study was approved by the Institutional Review Board of the Children's Hospital of Chongqing Medical University (File No. 2018-02, 2019-27-1). Informed consent was taken from children' $s$ guardians.

Open Access Statement: This is an Open Access article distributed in accordance with the Creative Commons Attribution-NonCommercial-NoDerivs 4.0 International License (CC BY-NC-ND 4.0), which permits the noncommercial replication and distribution of the article with the strict proviso that no changes or edits are made and the original work is properly cited (including links to both the formal publication through the relevant DOI and the license). See: https://creativecommons.org/licenses/by-nc-nd/4.0/.

\section{References}

1. Croisant S. Epidemiology of asthma: prevalence and burden of disease. Adv Exp Med Biol 2014;795:17-29.

2. von Mutius E, Smits HH. Primary prevention of asthma: 
from risk and protective factors to targeted strategies for prevention. Lancet 2020;396:854-66.

3. Bateman ED, Hurd SS, Barnes PJ, et al. Global strategy for asthma management and prevention: GINA executive summary. Eur Respir J 2008;31:143-78.

4. Asher I, Pearce N. Global burden of asthma among children. Int J Tuberc Lung Dis 2014;18:1269-78.

5. Nelson HS. Efficacy and safety of allergen immunotherapy in children. Ann Allergy Asthma Immunol 2006;96:S2-S5.

6. Murray CS, Poletti G, Kebadze T, et al. Study of modifiable risk factors for asthma exacerbations: virus infection and allergen exposure increase the risk of asthma hospital admissions in children. Thorax 2006;61:376-82.

7. Wang Y, Sun B, Zhen J, et al. Relationship between childhood asthma and atopy. J Pract Med 2002;(11):1163-5.

8. Gandhi VD, Davidson C, Asaduzzaman M, et al. House dust mite interactions with airway epithelium: role in allergic airway inflammation. Curr Allergy Asthma Rep 2013;13:262-70.

9. Green RM, Custovic A, Sanderson G, et al. Synergism between allergens and viruses and risk of hospital admission with asthma: case-control study. BMJ 2002;324:763.

10. Illi S, von Mutius E, Lau S, et al. Perennial allergen sensitisation early in life and chronic asthma in children: a birth cohort study. Lancet 2006;368:763-70.

11. Su KW, Chiu CY, Tsai MH, et al. Asymptomatic toddlers with house dust mite sensitization at risk of asthma and abnormal lung functions at age 7 years. World Allergy Organ J 2019;12:100056.

12. Haldar P, Pavord ID, Shaw DE, et al. Cluster analysis and clinical asthma phenotypes. Am J Respir Crit Care Med 2008;178:218-24.

13. Szefler SJ, Martin RJ, King TS, et al. Significant variability in response to inhaled corticosteroids for persistent asthma. J Allergy Clin Immunol 2002;109:410-8.

14. Tai A, Tran H, Roberts M, et al. Outcomes of childhood asthma to the age of 50 years. J Allergy Clin Immunol 2014;133:1572-8.e3.

15. Sherman RE, Anderson SA, Dal Pan GJ, et al. Real-World Evidence - What Is It and What Can It Tell Us? N Engl J Med 2016;375:2293-7.

16. Yan H, Siegel L, Breitbart S, et al. The Child \& Youth CompreHensIve Longitudinal Database for Deep Brain Stimulation (CHILD-DBS). Childs Nerv Syst 2021;37:607-15.

17. Yoon J, Eom EJ, Kim JT, et al. Heterogeneity of Childhood Asthma in Korea: Cluster Analysis of the
Korean Childhood Asthma Study Cohort. Allergy Asthma Immunol Res 2021;13:42-55.

18. Mahr TA, Lieberman JA, Haselkorn T, et al. Characteristics of Peanut Allergy Diagnosis in a US Health Care Claims Database (2011-2017). J Allergy Clin Immunol Pract 2021;9:1683-1694.e5.

19. Tan J, Peng X, Shu X, et al. Technical guidance for developing patient registry databases. Chinese Journal of Evidence-based Medicine 2019:771-8.

20. Dharmage SC, Perret JL, Custovic A. Epidemiology of Asthma in Children and Adults. Front Pediatr 2019;7:246.

21. Engelkes M, Janssens HM, de Ridder MA, et al. Time trends in the incidence, prevalence and age at diagnosis of asthma in children. Pediatr Allergy Immunol 2015;26:367-74.

22. Gough H, Grabenhenrich L, Reich A, et al. Allergic multimorbidity of asthma, rhinitis and eczema over 20 years in the German birth cohort MAS. Pediatr Allergy Immunol 2015;26:431-7.

23. Strachan DP, Cook DG. Health effects of passive smoking. 6. Parental smoking and childhood asthma: longitudinal and case-control studies. Thorax 1998;53:204-12.

24. Mastrorilli C, Posa D, Cipriani F, et al. Asthma and allergic rhinitis in childhood: what's new. Pediatr Allergy Immunol 2016;27:795-803.

25. Słabuszewska-Jóźwiak A, Szymański JK, Ciebiera M, et al. Pediatrics Consequences of Caesarean Section-A Systematic Review and Meta-Analysis. Int J Environ Res Public Health 2020;17:8031.

26. Khan DA. Allergic rhinitis and asthma: epidemiology and common pathophysiology. Allergy Asthma Proc 2014;35:357-61.

27. Murray CS, Foden P, Sumner H, et al. Preventing Severe Asthma Exacerbations in Children. A Randomized Trial of Mite-Impermeable Bedcovers. Am J Respir Crit Care Med 2017;196:150-8.

28. Virot E, Godet J, Khayath N, et al. Cluster analysis of indoor environmental factors associated with symptoms of mite allergy. Ann Allergy Asthma Immunol 2019;123:280-3.

29. Drury KE, Schaeffer M, Silverberg JI. Association Between Atopic Disease and Anemia in US Children. JAMA Pediatr 2016;170:29-34.

30. Yang F, Ghio AJ, Herbert DC, et al. Pulmonary expression of the human haptoglobin gene. Am J Respir Cell Mol Biol 2000;23:277-82.

31. Nguyen DT, Kit BK, Brody D, et al. Prevalence of high fractional exhaled nitric oxide among US youth with 
asthma. Pediatr Pulmonol 2017;52:737-45.

32. Dabbaghzadeh A, Tavakol M, Gharagozlou M. The Role of FENO in Comparison to Spirometry and ACT in Control of Children Asthma Symptoms. Iran J Allergy Asthma Immunol 2019;18:479-86.

33. Kanemitsu Y, Matsumoto H, Izuhara K, et al. Increased periostin associates with greater airflow limitation in patients receiving inhaled corticosteroids. J Allergy Clin
Immunol 2013;132:305-12.e3.

34. Fleming L, Murray C, Bansal AT, et al. The burden of severe asthma in childhood and adolescence: results from the paediatric U-BIOPRED cohorts. Eur Respir J 2015;46:1322-33.

(English Language Editor: K. Brown)

Cite this article as: Zhou J, Li W, Wen X, Zeng D, Lin J, Chen S, Zang N, Deng Y, Xie X, Ren L, Liu E. Establishing a patient registry study database of dust mite allergic asthma in children: design, methodology and preliminary exploration. Ann Transl Med 2021;9(12):993. doi: 10.21037/atm-21-2566 
Supplementary

Appendix 1 Questionnaire content

Name

Gender: male or female

date of birth

Time of first diagnosis of asthma

History of allergic rhinitis

Family history of allergy or asthma

Cigarette exposure

Delivery way

Premature labour or not

Medication (type, frequency and dose)

Asthma control score (c-ACT score)

Height and weight

History of food and drug allergy

whether have a history of other diseases

Result of skin prick tests to Der $p$ or Der $f$

What are the symptoms (wheezing, cough, shortness of breath, chest tightness, et al.) 